\title{
Loneliness and Major Depressive Disorder in the Elderly with a History of Suicidal Ideation or Attempt: A Comment on "Therapist-Guided Internet- Based Treatments for Loneliness" by Käll et al.
}

\author{
Hiu Ching Siu ${ }^{a}$ Shwu-Hua Lee ${ }^{b, c}$ Josephine Sheron Au ${ }^{d}$ Alfred Pak-Kwan Lo ${ }^{a}$ \\ Chih-Mao Huang ${ }^{e, f}$ Yun-Fang Tsaig, h Tatia Mei-Chun Lee ${ }^{i, j}$ Ho-Ling Liu ${ }^{k}$ \\ Chemin Lin ${ }^{c, l, m}$ Chui-De Chiu ${ }^{a}$
}

\begin{abstract}
aDepartment of Psychology, The Chinese University of Hong Kong, Hong Kong SAR, China; 'bepartment of Psychiatry, Linkou Chang Gung Memorial Hospital, Taoyuan, Taiwan; 'College of Medicine, Chang Gung University, Taoyuan, Taiwan; ${ }^{\mathrm{d} C h i l d}$ and Adolescent Psychiatry Division, Department of Psychiatry, McLean Hospital, Harvard Medical School, Boston, MA, USA; 'Department of Biological Science and Technology, National Yang Ming Chiao Tung University, Hsinchu, Taiwan; ${ }^{f}$ Center for Intelligent Drug Systems and Smart Bio-devices (IDS2B), National Yang Ming Chiao Tung University, Hsinchu, Taiwan; ${ }^{9}$ School of Nursing, College of Medicine, Chang Gung University, Taoyuan, Taiwan; 'Department of Nursing, Chang Gung University of Science and Technology, Taoyuan, Taiwan; 'Laboratory of Neuropsychology and Human Neuroscience, The University of Hong Kong, Hong Kong SAR, China; 'State Key Laboratory of Brain and Cognitive Sciences, The University of Hong Kong, Hong Kong SAR, China; ' Department of Imaging Physics, University of Texas MD Anderson Cancer Center, Houston, TX, USA; 'Department of Psychiatry, Keelung Chang Gung Memorial Hospital, Keelung, Taiwan; ${ }^{\mathrm{m} C o m m u n i t y}$ Medicine Research Center, Keelung Chang Gung Memorial Hospital, Keelung, Taiwan
\end{abstract}

Käll et al. [1] demonstrated that loneliness, a prevailing risk factor of mental and physical health problems, can improve with psychological interventions. Despite notable differences between internet-based cognitive behavioral therapy and interpersonal therapy, both had medium-to-large within-group effect sizes 4 months post-intervention. This encouraging finding indicates that clinical management of loneliness in addition to treatment for depression and anxiety is a possible avenue to prevent elderly suicide, as we demonstrated in our study that loneliness and mood disorder insight [2] were proximal to state suicidality among the elderly.

The study aims to examine the role psychological factors play in the link of elderly depression and suicidality. While major depressive disorder has been established as

karger@karger.com www.karger.com/pps

Karger"
(C) 2021 The Author(s)

Published by S. Karger AG, Basel

This is an Open Access article licensed under the Creative Commons Attribution-NonCommercial-4.0 International License (CC BY-NC) (http://www.karger.com/Services/OpenAccessLicense), applicable to the online version of the article only. Usage and distribution for commercial purposes requires written permission. a leading predictor of suicide, existing theories assert that it may only be relevant due to its impact on certain psychological factors [3]. For instance, enhanced distress, reduced reward, and capability of suicide may mediate the relationship between depression and suicide $[4,5]$. As such, clarifying the psychological factors proximal to current suicidality should benefit the clinical management of suicidality among patients with depression.

The hypothesis was tested in a sample of Taiwanese elderly outpatients with major depressive disorder (MDD; $n=120$, age $=66.78 \pm 5.67$ years, $18 \%$ male, years of education $=8.17 \pm 3.62)$ and healthy controls $(n=20$, age $=$ $69.05 \pm 5.38$ years, $40 \%$ male, years of education $=11.35$

H.C. Siu and S.-H. Lee contributed equally to this work. 
$\pm 4.17)$. The Structural Clinical Interview of the 5th edition of the Diagnostic and Statistical Manual of Mental Disorders, and the Mini-International Neuropsychiatric Interview were conducted, respectively, to make the MDD diagnosis in patients and to ensure the absence of major psychiatric disorders in the healthy participants. Item No. 3 of the Hamilton Rating Scale for Depression (HAM-D) was used to group the participants diagnosed with depression based on their history of suicidal behaviors (ideator, $n=42$; attempter, $n=30$; depressed control, $n=48)$. No demographic differences were evident among the clinical groups. For the attempter group, the mean age of the first attempt was 53 years $(S D=13$, range 20-78). The average number of previous suicidal attempts was $2.26(\mathrm{SD}=1.58$, range $1-6)$.

Validated Chinese versions of clinician- and self-rating scales were used to evaluate psychiatric dysfunction and psychological factors. State suicidality was evaluated by the Beck Scale for Suicide Ideation (BSS). Depression and anxiety were assessed by the HAM-D and the Hamilton Anxiety Rating Scale (HAM-A). Enhanced distress was assessed by the Mood Disorder Insight Scale and the neuroticism subscale in the Eysenck Personality Questionnaire (EPQ). Reduced reward was captured by the Apathy Evaluation Scale, the Short-Form Health Survey (which measures quality of life), the extraversion subscale of the EPQ, and the UCLA Loneliness Scale. Impulsivity was measured by the Barratt Impulsivity Scale. The following variables that indicate cognitive functioning were used as control variables in the analysis [6]. These include global cognition assessed by the Mini-Mental State Examination and executive controls indicated by a composite score based on the Digit Span, Letter-Number Sequencing, Digit Symbol Coding, and Category Fluency tests.

State suicidality were significantly correlated with all but two (i.e., neuroticism and apathy) across depression and anxiety, enhanced distress, reduced reward, and impulsivity (from the smallest effect size with impulsivity, Cohen's $d=0.47$, to the largest with loneliness, $d=0.95$ ). To identify the unique effect of each variable on state suicidality, multiple regression analysis was performed, with depression and anxiety, the psychological factors, cognitive functioning, and demographics serving as independent variables simultaneously. Two models were constructed separately for depression and anxiety to prevent the collinearity. The effects of depression and anxiety ( $\beta s=0.10$ and $0.17, t s=0.74$ and 1.33 , $p s=0.46$ and 0.19$)$ became insignificant when the psychological factors were considered. Among the psychological factors, the effect of loneliness (in the models of depression and anxiety, $\beta \mathrm{s}=$ 0.44 and $0.40, t s=2.94$ and $2.71, p s<0.01)$ and mood disorder insight $(\beta \mathrm{s}=0.28$ and $0.29, t \mathrm{~s}=2.32$ and $2.38, p \mathrm{~s}=$ $0.02)$ remained significant.

Some limitations should be noted. Regarding loneliness, the results did not differentiate a lack of social contact per se [7] from perceived rejection [8]. Whether or not an internalized rejection is sufficient for the sense of disconnectedness in suicide remains to be investigated [ 9 , 10]. Second, attempters recruited in an elderly sample may not be representative of populations that attempt suicide at a younger age as the mean age of first suicide attempt was 53 years. Nonetheless, in light of the recent findings by Käll et al. [1], this study suggests that a targeted intervention on loneliness should be considered for the elderly with depression and a history of suicidality.

\section{Acknowledgements}

We thank Lack Liu for his assistance in participant recruitment and data collection, and participants as well as family members for their kind participation.

\section{Conflict of Interest Statement}

The authors have no conflicts of interest to declare.

\section{Funding Sources}

This research was supported by two grants (No. NMRPG3G6031/32 and No. NMRPG3J0121) from the National Science Council, Taiwan, to S.-H. Lee; by grants from the Research Grant Council, Hong Kong SAR (GRF, No. 14612519), from the Social Science Panel from The Chinese University of Hong Kong (No. 4052207 and No. 4052174), from the Brain and Behavior Research Foundation and the Families for Borderline Personality Disorder Research, USA (2018 NARSAD Young Investigator Grant, No. 27180) to C.-D. Chiu.

\section{Author Contributions}

S.-H. Lee and C.-D. Chiu jointly conceived the initial research idea. H.C. Siu and C.-D. Chiu conducted the literature review and developed the conceptual framework with J.S. Au. S.-H. Lee, and C. Lin contributed to study design and data collection together with C.-M. Huang, Y.-F. Tsai, T. M.-C. Lee, and H.-L. Liu. C.-D. Chiu performed the data analysis and interpreted the data with A.P.-K. Lo and H.C. Siu. H.C. Siu and C.-D. Chiu prepared the first draft in conjunction with J.S. Au and A.P.-K. Lo. All authors participated in revisions of the manuscript and approved the final version for submission. 


\section{References}

1 Käll A, Bäck M, Welin C, Åman H, Bjerkander R, Wänman $M$, et al. Therapist-guided internet-based treatments for loneliness: a randomized controlled three-arm trial comparing cognitive behavioral therapy and interpersonal psychotherapy. Psychother Psychosom. 2021 Aug;90(5):351-8. http://dx.doi. org/10.1159/000516989.

2 Buchman-Wildbaum T, Váradi E, Schmelowszky Á, Griffiths MD, Demetrovics Z, Urbán R. The paradoxical role of insight in mental illness: the experience of stigma and shame in schizophrenia, mood disorders, and anxiety disorders. Arch Psychiatr Nurs. 2020 Dec;34(6):449-57.

3 Klonsky ED, May AM, Saffer BY. Suicide, suicide attempts, and suicidal ideation. Annu Rev Clin Psychol. 2016 Mar;12:307-30.
4 Chu C, Walker KL, Stanley IH, Hirsch JK, Greenberg JH, Rudd MD, et al. Perceived problem-solving deficits and suicidal ideation: evidence for the explanatory roles of thwarted belongingness and perceived burdensomeness in five samples. J Pers Soc Psychol. 2018 Jul;115(1):137-60. http://dx.doi. org/10.1037/pspp0000152.

5 O'Connor RC, Kirtley OJ. The integrated motivational-volitional model of suicidal behaviour. Philos Trans R Soc Lond B Biol Sci. 2018 Sep;373:20170268. http://dx.doi.org/10.1098/ rstb.2017.0268

6 Miranda R, Gallagher M, Bauchner B, Vaysman R, Marroquín B. Cognitive inflexibility as a prospective predictor of suicidal ideation among young adults with a suicide attempt history. Depress Anxiety. 2012 Mar;29(3): 180-6.
7 Tsai AC, Lucas M, Kawachi I. Association between social integration and suicide among women in the United States. JAMA Psychiatry. 2015 Oct;72(10):987-93.

8 Shahar G, Rogers ML, Shalev H, Joiner TE. Self-criticism, interpersonal conditions, and biosystemic inflammation in suicidal thoughts and behaviors within mood disorders: a bio-cognitive-interpersonal hypothesis. J Pers. 2020 Feb;88(1):133-45. http:// dx.doi.org/10.1111/jopy.12446.

9 Chiu CD, Siu CY, Ng HC, Baldwin MW. Visuospatial perspective shifting and relational self-association in dispositional shame and guilt. Conscious Cogn. 2021 Jul;92:103140.

10 Chiu CD, Ho HL, Tollenaar MS. Relational self-evaluations in dissociation: implicit selfrejection? Psychol Trauma. 2021 Feb. http:// dx.doi.org/10.1037/tra0001017. 